\title{
A Survey on Scheduling Algorithms for Wireless Sensor Networks
}

\author{
Sumit Kumar \\ M.Tech Scholar \\ Department of Computer Science and \\ Engineering, National Institute of Technology, \\ Hamirpur (H.P.), India
}

\author{
Siddhartha Chauhan \\ Assistant Professor \\ Department of Computer Science and \\ Engineering, National Institute of Technology, \\ Hamirpur (H.P.), India
}

\begin{abstract}
Wireless sensor networks(WSN) have wide range of application such as traffic analysis, environmental monitoring, industrial process monitoring, and tactical systems. Large-scale wireless sensor networks are expected to play increasingly important role in future civilian and military application. Designing of MAC layer protocol for wireless sensor network is a challenging task due to limited battery power and limited bandwidth. Time Division Multiple Access Protocol solves both problems at the level of MAC layer. Various scheduling method for TDMA protocol with different objective have been proposed for wireless sensor networks. In this paper, we first outline the sensor network properties that are crucial for the design of TDMA protocols and then, we describe several TDMA protocols which are proposed for sensor networks. Finally, we point out open research issue with regard to TDMA protocols.
\end{abstract}

\section{General Terms}

Wireless Sensor Network, MAC Protocol

\section{Keywords}

TDMA, MAC Layer, Scheduling, Latency, Wireless sensor network(WSN)

\section{INTRODUCTION}

Wireless sensor networks usually contain thousands of sensors, which are randomly and densely deployed. Each sensor has a light weight and a low cost with three technologies of sensing, on-board processing and transmission. Sensor nodes have limited battery power which leads to limited coverage and communication range[1].

Most of the applications in wireless sensor networks involve primarily data aggregation in which sensor node periodically produced data and transmitting to the sink node through the aggregated node where continuous queries are posed and processed. But data aggregations in WSN have two main issues: First, save energy in battery powered sensor and second, fast and efficient query response are essential to network performance and maintenance. In sensor node, both sensor element and processing element consume constant and low power[2]. Energy used by the transceiver is variable and very high in comparison to sensing and processing energy. The power consumed in the transmission depends upon the network topology, MAC layer protocol, routing algorithms, data fusion and cache memory in sensor node. In this paper, we are describe the advantage and disadvantage of several TDMA protocols which is used in MAC layer.

In wireless sensor network, during the data aggregation any node listen the channel every time even if data is not placed on the channel that is called ideal listening as nodes do not know when data will come from its child nodes. But TDMA scheduling removes the idle listening because every node has a fixed time slot for transmitting and receiving. Every node after receiving or transmitting data goes in sleep mode or processing the data and this technique saves battery power.

\section{TDMA- PROTCOL-RELATED SENSOR NETWORK PROPERTIES}

In WSN, saving the battery power is most important issue, by which we can increase the lifetime of the network. Sensor nodes are assumed to be dead when they are out of battery. Another issue is fast and efficient query response, so that every change in the environment can be detected immediately. On the bases of above characteristics, the proposed TDMA protocol must be energy efficient by reducing the potential energy wastes (presented below) and send the senses data to the sink without further delay. TDMA protocols reduce the data retransmission because collision does not occur in TDMA protocol. The types of communication patterns that are observed in sensor network applications should be investigated, since these patterns determine the behavior of the sensor network traffic that has to be handled by TDMA protocol.

\section{REASONS OF ENERGY WASTE}

In wireless sensor network, if network has limited number of non overlapping channels than a different channel is not assigned to every node on the data aggregation tree. Hence data aggregation introduces data retransmission which is caused by co-channel interference from neighboring sensor nodes and extra battery power is required for retransmission of data[3]. When a node receive more than one packet at the same time then these packet collide with each other. All packets that cause the collision have to be discarded and retransmissions of these packets are required, which increases the energy consumption. One of the major sources of energy waste is idle listening. An ideal listening occurs when a node listen an ideal channel in order to receive possible traffic. The third reason for energy waste is overhearing [2]. Overhearing occurs when a node receives packets which are going to other destination. The fourth reason of energy wastage is transmitted when redundant data packet or redundant data to the destination. The one another 
reason for energy wastage is overemitting [2]. Overemitting is caused by the transmission of a data packet when the destination is not ready. The above factors of energy wastage do help to design an efficient protocol.

\section{NETWORK TOPOLOGY COMMUNICATION PATTERN}

AND

In WSNs', Networks are divided into three categories, first is tree based[4], second is cluster based[5], and third is hybrid topology. In tree based structure, the root of tree is called sink node. At the sink, monitored data by the sensor node is collected where continuous queries are posed and processed. In WSN tree data flows from leaf nodes to sink node through the intermediate nodes. These intermediate nodes are called aggregator nodes. These aggregator nodes receive data from its child nodes. Aggregator nodes process and add their own monitored data and send to its parent node. In clustered approach, every cluster has a cluster head and the entire node in this cluster transmit data to the cluster head $(\mathrm{CH})$. This $\mathrm{CH}$ transmits data to $\mathrm{CH}$ of another cluster which is nearest to sink node or to sink node.

Kulkarni define three types of communication patterns in wireless sensor networks [6]: first is broadcast, second is convergecast, and third is local gossip. In broadcasting, any information is transmitted to all sensor nodes. It is used by a base station or by the sink node. If any updates occur in sensor program, queries of sensor query-processing architectures, or control packets which is used by the whole system are transmitted by the base station or sink. In local communication pattern, a node communicates to its neighbor nodes or they are in its communication range. In convergecast communication pattern, groups of specified sensor nodes they have need of communication transmit data to a sensor node. The destination node could be a cluster head, a data fusion centre, or a base station. In cluster based networks, cluster heads communicate with their members, these members may be all the neighbors of cluster head or a subset of neighbors of cluster head. This type of communication pattern is called multicast communication pattern.

\section{PROPERTIES OF A WELL-DEFINED TDMA PROTOCOLS}

To design a efficient TDMA protocol for wireless sensor network the following attributes must be considered. Firstly, TDMA protocol should be energy conserving protocol, because energy is a limited resource in wireless sensor network. Secondly, another attribute of wireless sensor network is scalability and adaptability to change. If any change in network size, node density, and topology, should be handled effectively by protocols. Fast and efficient query response is another essential attribute for network performance in wireless sensor networks. Co-channel interference is also a problem, hence interference should be minimized where ever possible. There are other attributes such as latency, throughput, and bandwidth utilization which may be considered secondary for application of sensor networks.

\section{PROPOSED TDMA PROTOCOLS}

In this section, a wide range of TDMA protocols defined for sensor networks are described briefly with their advantages and disadvantages.

\subsection{Energy-Efficient Wake-Up Scheduling MAC Protocol}

This TDMA protocol uses energy efficient Wakeup scheduling(EEWS) algorithm [7] for sensor nodes which reduces the number of wake-up. Wake-up means, when any node receives or transmits data then it comes in wakeup mode. In this protocol, to reduce the energy cost, the activities of a subset of sensors are schedule in one bundle, in which one node wakeup at most twice: once for receiving data from its all child nodes and once for transmission the data to its parent node.

In homogeneous network, Let $\mathrm{C}_{\mathrm{T}}\left(\mathrm{V}_{\mathrm{i}}\right)$ denote the set of children nodes of node $\mathrm{V}_{\mathrm{i}}$ in data gathering tree $\mathrm{T}$. Then we say that $\mathrm{C}_{\mathrm{T}}\left(\mathrm{V}_{\mathrm{i}}\right)$ constitutes a virtual cluster $\mathrm{C}_{\mathrm{i}}$. The weight $\mathrm{W}_{\mathrm{i}}$ is define the total number of time slots that node $\mathrm{V}_{\mathrm{i}}$ should wake up to receive the data from its children in the data gathering tree. Instead of scheduling the transmitting time slots for each individual child node of node $\mathrm{V}_{\mathrm{i}}$, we schedule a chunk of consecutive $\mathrm{W}_{\mathrm{i}}$ time slots to the cluster $\mathrm{C}_{\mathrm{i}}$ of these child nodes All the nodes send their data in this time period and parent receives the data atthe same time period, as shown in Figure 1. So node $V_{i}$ will wakeup twice: once for receiving data from its children because its children have consecutive time slots and other for transmission its data to parent node.

In Heterogeneous networks, sensor nodes divide in buckets, $\mathrm{i}^{\text {th }}$ bucket contains all sensor node which have the interference radius within $\left(2^{i-1} R_{\min }, 2^{i} R_{\min }\right)$. Here $R_{\min }$ is the minimum interference radius of sensor nodes in network. Nodes in Cluster or bucket have consecutive time slots for a chunk. All the nodes send their data in this time period and parent receive the data at same time period. So node $V_{i}$ will wakeup twice: once for receiving data from its children because its children have consecutive time slots and other for transmission its data to parent node.

Advantage: First advantage of this protocol is that it is energy conserving. Energy saved by reducing the state transition from sleeping to wakeup state and from wakeup to sleeping state. This also dramatically reduces the cost of the clock synchronization. This scheduling protocol also reduces time delay because slots are reused.

Disadvantage: The main disadvantage with this protocol is finding the clusters which have contention with each other and allot different time slot to them. Due to slot reuse co-channel interference can be occur if two contended clusters use same time slot. 


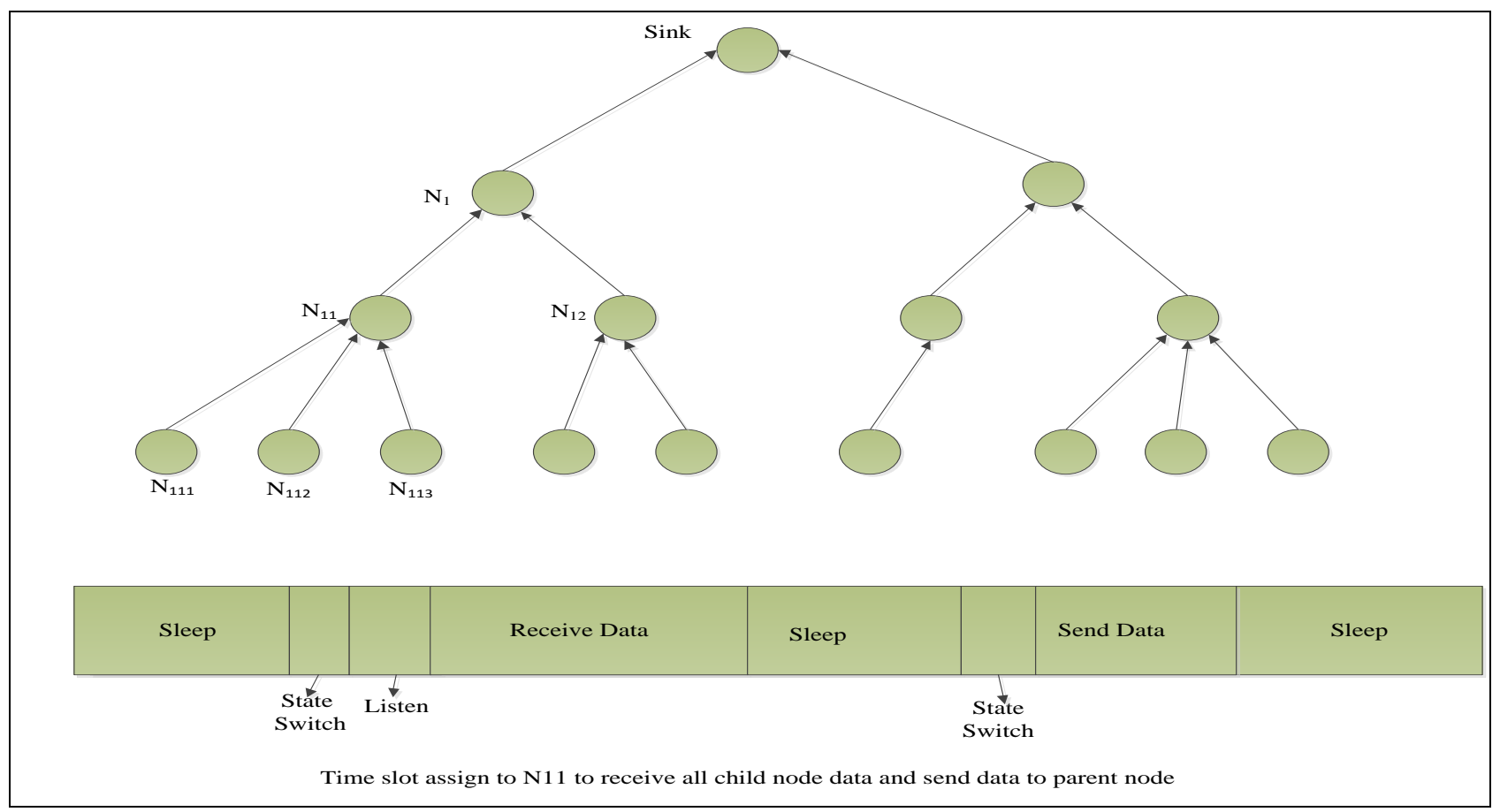

Fig 1: Time slot assign to a node in Energy-Efficient Wake-Up Scheduling for receiving and sending data[7]

\subsection{Minimum Delay Scheduling TDMA Protocol}

Minimum Delay scheduling algorithm (MDS)[8] reduced the time delay in clustered wireless sensor network. This scheduling algorithm incorporates the slot reuse concept and slot reuse concept significantly reduces the end-to-end latency without a penalty in the energy efficiency. In this protocol, derived a relationship between the delay incurred by a data packet at each backbone network node (along its path to the sink) and the TDMA frame length $M$ so that, by reducing the frame length through slot reuse, the delay is minimized. For this analysis, determines the node delay from the instant the first bit arrives at the node until the time the last bit exits the node. The end to end delay is the sum of the nodal delays along a path to the sink, plus the associated propagation delays which are negligible due to short distances between the backbone nodes. Hence, minimizing the TDMA frame length also minimizes the end-toend delay.

Next, this algorithm applies the slot reuse concept to achieve a reduction in TDMA frame length. That is, a slot already assigned to a link can be reused at another link provided that the interference between the two links is below a specified threshold. In slot reuse, the first step is to determine the number of slots required by each link in the backbone network and the virtual link in each cluster. For a backbone link $(i, j)$ with flow $f$, maximum data rate $R$ (both in bits per second), and per-hop packet loss rate $\mathrm{p}$, the slots needed (each of length $\mathrm{t}$ ) per second are $M=\left(f /\left(R^{*} t(1-p)\right)\right)$. This algorithm uses a simple incremental heuristic to calculate the TDMA schedule that has the shortest length. The heuristic can start from any of the backbone nodes (CH or gateway), assigning slots first to the virtual link (if the node is a $\mathrm{CH}$ ), and then, to all the outgoing links of the node, as shown in Fig. 2. Before assigning slots to any succeeding nodes, the algorithm checks all the links that are previously assigned with slots to make sure no conflicts exist. Three criteria that guarantee the conflict free communication is 1) Node $i$ is a sender of slot $\mathrm{s}$; thus, slot $\mathrm{s}$ cannot be assigned to any other links of node $i$. 2) Node $\mathrm{i}$ is a receiver of slot $\mathrm{s}$; thus, slot $\mathrm{s}$ cannot be assigned to any other links of node $i .3)$ Slot $\mathrm{s}$ is assigned to link $(\mathrm{m}, \mathrm{n})$; thus, cannot be assigned to link $(i, n)$.

Advantage:- This algorithm uses the cross layer optimization model to achieve energy efficiency with specified link reliability and bandwidth constraints. Minimum Delay TDMA Scheduling Algorithm reduced the time delay at the node and new data will collect at the sink node very rapidly which leads fast and efficient query response.

Disadvantage:- The major disadvantage of this protocol is cochannel interference if two node within the interference get same slot. Co-channel interference leads the data retransmission which reduces the battery life time. And another disadvantage is that the state transition is more which consume extra energy.

\subsection{Minimum Delay Scheduling TDMA Protocol}

A distributed Algorithm generates a collision free schedule for data aggregation in wireless sensor networks. The time latency of the aggregation schedule generated by this algorithm is minimized using a greedy strategy. This distributed aggregation scheduling algorithm [9], named DAS, consists of two phases. One is to construct a distributed aggregation tree and another 


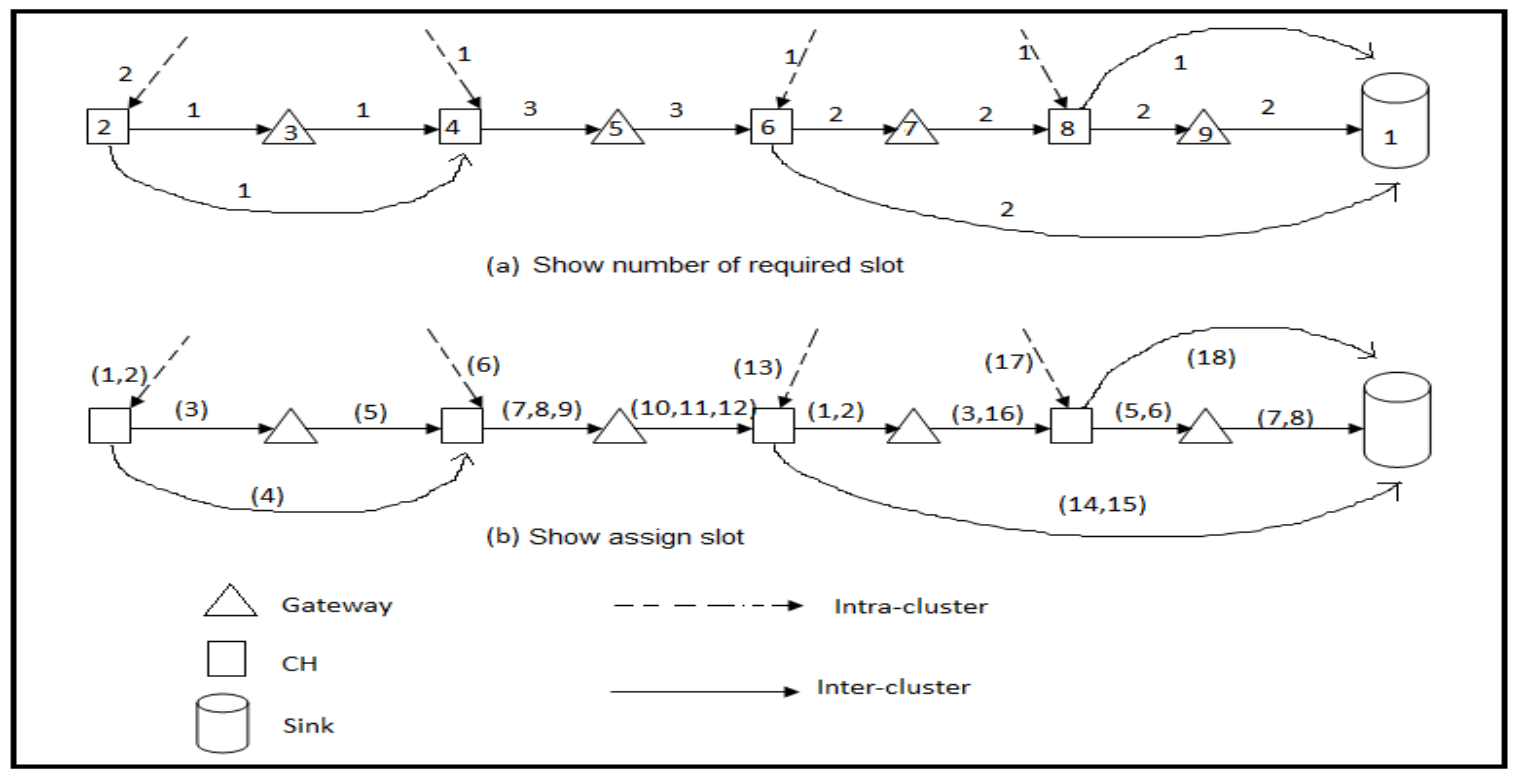

Fig 2: Slot assignment using Minimum Delay Scheduling algorithm [8].

one is to perform the distributed aggregation scheduling.

A distributed approach of constructing a CDS has been proposed by Wan et.al. in [10]. In this algorithm, an MIS of the network is constructed first which is constructed by the all black node as shown in Fig. 3 and then a dominating tree is constructed by concatenating MIS nodes and the other nodes. The rest nodes are gray and white. The white nodes are leaves in the aggregation tree and each gray node plays the role of connecting two black nodes. The root of the aggregation tree is a black node and all its neighbors are its children that can only be white or gray nodes. For each gray node, its children must be black nodes. For each black node, its children must be either white or gray.

The second phase of DAS is to determine the schedules for all the nodes in a sensor network in a distributed manner to solve the distributed aggregation scheduling problem. In distributed data aggregation scheduling each node is set to one state of NOT-READY (NRY), READY (RY), WAIT0, WAIT1, SCHEDULE-COMPLETED (SC) and SLEEP at every time instant, as shown in Fig. 3. During the scheduling, each node works according to the automaton shown in Figure 3. Initially all non-leaf node is in NRY state and all leaf node is in RY state. When all children of a node finish their transmission operation then the parent node will go in RY state. When a node u comes into RY state, u sends a MARK message containing u's ID and slot $\mathrm{K}$ to request for the feedback messages from all its competitors and turns into state WAIT0. When a node $\mathrm{u}$ in WAIT0 gets all feedback messages, it checks if its ID is larger than IDs of all the nodes in R1(u), where R1(u) is set of all ready nodes, then $u$ calls a procedure to fix its schedule and send a SCH-COMPLETE message consisting of u's ID and $\mathrm{K}$ to all nodes in R1(u), else it goes to WAIT1 state, waiting for scheduling later. A node $\mathrm{u}$ in WAIT1 state must wait for being scheduled until all the ready nodes with larger IDs are scheduled. Upon receiving a new SCH-COMPLETE message, a node in WAIT1 checks if its ID is larger than Ids of all the nodes in R1(u). If so, it calls a procedure to fix its schedule, else it keeps waiting in WAIT1 state. When the root comes into RY state, the algorithm ends and all the nodes complete their schedules.

Advantage: Some Networks topologies are changed due to node failures or due to moving the node. This algorithm is good for this type topology because it reduces the cost of recomputed a schedule and disseminate. And other advantages are less time latencies, collision free and inherently efficient algorithm.

Disadvantage: This algorithm takes energy to sends MARK message to all competitor but energy is limited resource in wireless energy. And energy wastage while nodes will be in waits state because these are not in sleeping state.

\subsection{Traffic-Adaptive MAC Protocol}

Traffic-Adaptive MAC protocol (TRAMA)[11] is a TDMA based algorithm proposed to increase the TDMA utilization in energy efficient manner. TRAMA uses a distributed election algorithm which is based on the traffic information and find the time slot for transmitting data within each two-hop neighborhood according to traffic information. This algorithm, time is divided into random-access and scheduled-access (transmission) periods. Random access period refers signaling slots, which are used to obtain consistent two-hop topology information across all nodes. Channel access is contentionbased within that period. Transmission slots are used for collision-free data exchange and also for schedule propagation.

TRAMA is divided into three part, first part is Neighbor Protocol (NP), second part is Schedule Exchange Protocol (SEP), and third is the Adaptive Election Algorithm(AEA). NP propagates one hop neighbor information during the random access period by using signaling slot to obtain consistent topology information and perform channel acquisition. 


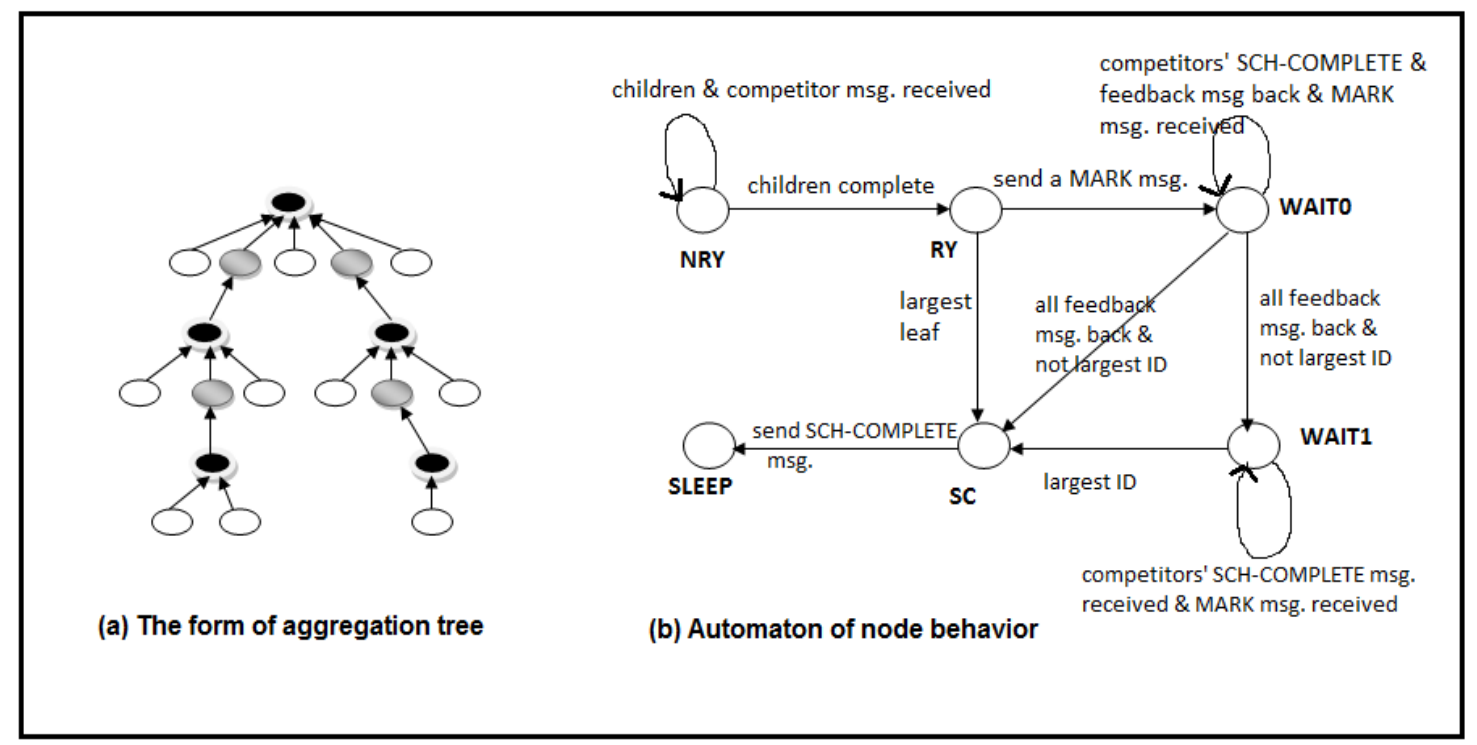

Fig 3: Distributed Data Aggregation Scheduling [9]

Nodes use SEP to exchange traffic information and also schedules with neighbors. A basic assumption is that, with the information passed by the application layer, the MAC layer can calculate the transmission duration needed, which is denoted as SCHEDULE_INTERVAL. Then, at time $t$, the node calculates the number of slots for which it will have the highest priority among two-hop neighbors within the period $[t, t+$ SCHEDULE_INTERVAL]. The node announces the slots it will use as well as the intended receivers for these slots with a schedule packet. Additionally, the node announces the slots for which it has the highest priority but it will not use. The schedule packet indicates the intended receivers using a bitmap whose length is equal to the number of its neighbors. Bits correspond to one-hop neighbors ordered by their identities. Since the receivers of those messages have the exact list and identities of the one-hop neighbors, they find out the intended receiver.

AEA selects the transmitter and receiver for a slot by using the information gathered from the NP and SEP and achieves collision free communication. If a node do not have any data to transmit then it may give up its time slot, and another node can use this time slot to transmit data. When the vacant slots are announced, potential senders are evaluated for reuse of those slots. Priority of a node on a slot is calculated with a hash function of node's and slot's identities. Delays in TRAMA are found to be higher, as compared to those of Contention-based protocols, due to a higher percentage of sleep times.

Advantages- In this algorithm, time slot which is not used by any node, is used by the other node. Higher percentage of sleep time and less collision probability are achieved, as compared to CSMA-based protocols. Since the intended receivers are indicated by a bitmap, less communication is performed for the multicast and broadcast types of communication patterns, compared to other protocols.

Disadvantages- Transmission slots are set to be seven times longer than the random-access period [11]. However, all nodes are defined to be either in receive or transmit states during the random-access period for schedule exchanges. This means that without considering the transmissions and receptions, the duty cycle is at least 12.5 percent, which is a considerably high value. For a time slot, every node calculates each of its two-hop neighbors' priorities on that slot. This calculation is repeated for each time slot, since the parameters of the calculation change with time. Hence passing massages are more which consumed additional energy.

\subsection{DMAC Protocol}

The data forwarding interruption problem which is exist in implicit adaptive duty cycle technique because overhearing range is limited in wireless sensor node. In DMAC[12] Protocol, a staggered active/sleep scheduling is done to solve this problem and enable continuous data forwarding on the multihop path. The principal aim of DMAC is to achieve very low latency for convergecast communications, but still is energy efficient.

In staggered active/sleep scheduling, RTS and CTS control packets is not used because they give unnecessary overhead but ACK packet and data retransmission are used to recover the lost data packet. To reduced collision during the Transmission(Tx) period of nodes on the same tree level, every node backs off for a period (BP) plus a random time within a contention window before packet transmission. In contention window, the size of one slot is only enough to transmit one packet. When a node receives a data packet during $\operatorname{Receiving}(\mathrm{Rx})$ period then it wait for a small time period (SP) and then send ACK. packet back to the sender. The sending and receiving slot length is $(\mathrm{BP}+\mathrm{CW}$ + DATA + SP + ACK) where CW is the fixed contention window. Local synchronization is needed in DMAC since a node needs to be aware of its neighbors' schedule.

Convergecast is the most frequent communication pattern observed within sensor networks. Unidirectional paths from 


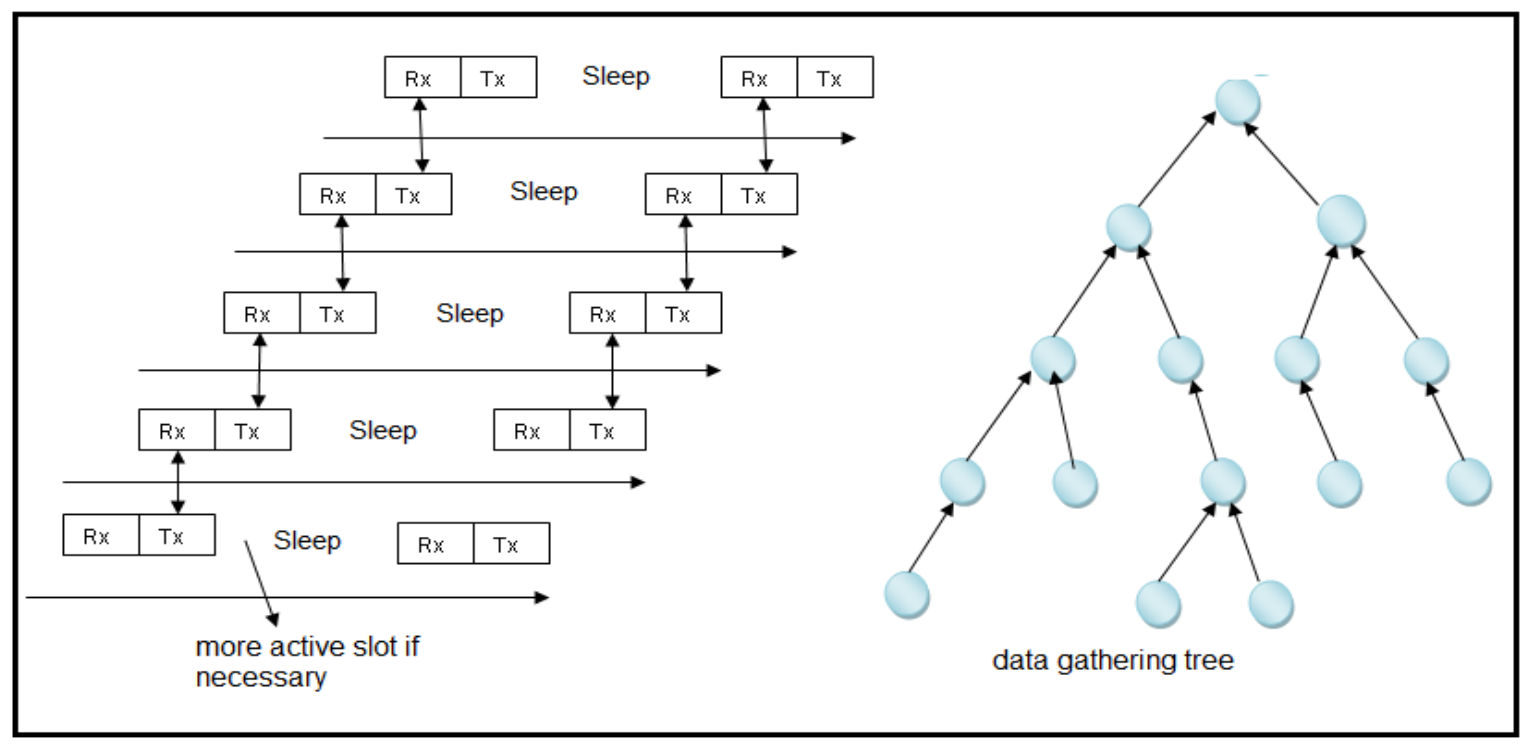

Fig 4: A data gathering tree and its DMAC implementation [12].

sources to the sink could be represented as data-gathering trees. DMAC could be summarized as an improved Slotted Aloha algorithm in which slots are assigned to the sets of nodes based on a data gathering tree, as shown in Figure 4. Hence, during the receive period of a node, all of its child nodes have transmit periods and contend for the medium. Low latency is achieved by assigning subsequent slots to the nodes that are successive in the data transmission path.

Advantages - DMAC achieves very good latency compared to other sleep/listen period assignment methods. The latency of the network is crucial for certain scenarios; hence DMAC can be used for such application.

Disadvantages - Collision avoidance methods are not utilized; hence, when a number of nodes that have the same schedule (the same level in the tree) try to send to the same node, collisions will occur. This is a possible scenario in event triggered sensor networks. Besides, the data transmission paths may not be known in advance, which precludes the formation of the datagathering tree.

\section{OPEN ISSUE AND CONCLUSION}

Table 1 gives a comparison of the TDMA protocols which are investigated above. "Time Synchronization Needed" refers to time synchronization achieved externally, "Co-channel Interference" refers to interference produced by neighbor node, "Time Latency" is the time difference between time at which sensor node senses sample and the time when this data sample reaches at the sink, "Adaptivity to Changes" indicates the ability to handle topology changes.

Table 1. Comparison of TDMA protocols.

\begin{tabular}{|c|c|c|c|c|c|}
\hline $\begin{array}{l}\text { Scheduling } \\
\text { Protocol }\end{array}$ & Time Latency & $\begin{array}{l}\text { Co-channel } \\
\text { Interference }\end{array}$ & $\begin{array}{l}\text { Time } \\
\text { Synchronization } \\
\text { Needed }\end{array}$ & $\begin{array}{l}\text { Comm. } \\
\text { pattern } \\
\text { support }\end{array}$ & $\begin{array}{l}\text { Adaptivity to } \\
\text { changes }\end{array}$ \\
\hline EEWS & High & yes & $\begin{array}{l}\text { Reduced cost of } \\
\text { Synchronization }\end{array}$ & All & Good \\
\hline MDS & Low & Yes & Yes & All & Good \\
\hline DAS & Low & No & Yes & All & Good \\
\hline TRAMA & High & No & Yes & All & Good \\
\hline DMAC & Low & No & Yes & Convergecast & Weak \\
\hline
\end{tabular}


Although there are various TDMA based MAC layer protocols proposed for sensor networks, there is no protocol accepted as a standard. One of the reasons for this is that the MAC protocol choice will, in general, be application dependent, which means that there will not be one standard MAC for sensor networks. Another reason is the lack of standardization at lower layers (physical layer) and the (physical) sensor hardware.

TDMA has a natural advantage of collision-free medium access but in some protocols this leads to interference due to slot reuse. Time synchronization is required in these protocols because of clock drift problem. Adaptation to topology changes is another difficulty faced by TDMA system as these changes are caused by insertion of new nodes, exhaustion of battery capacities, broken links due to interference, the sleep schedules of relay nodes, and scheduling caused by clustering algorithms. The slot However, it is not easy to change the slot assignment within a decentralized environment for traditional TDMA, since all nodes must agree on the slot assignments.

Common wireless networking experience also suggests that link-level performance alone may provide misleading conclusions about the system performance. A similar conclusion can be drawn for the upper layers as well. Hence, the more layers contributing to the decision, the more efficient the system can be. For instance, the routing path could be chosen depending on the collision information from the medium access layer. Moreover, layering of the network protocols creates overheads for each layer, which causes more energy consumption for each packet. Therefore, integration of the layers is also a promising research area that needs to be studied more extensively.

\section{REFERENCES}

[1] Min Ding, Xiuzhen Cheng, and Guoliang Xue, "Aggregation Tree Construction in Sensor Networks", IEEE, 2003, pp 2168-2172.

[2] Ilker Demirkol, Cem Ersoy, and Fatih Alagöz, "MAC Protocols for Wireless Sensor Networks: A Survey", IEEE Communications Magazine, April 2006, pp 115121.

[3] H.-H. Yen, and C.-L. Lin, "Integrated channel assignment and data aggregation routing problem in wireless sensor networks", IEEE, In Special Issue on Wireless Ad-Hoc Networks, 2009.
[4] Xingbo Yu, Sharad Mehrotra, and Nalini Venkatasubramanian, "Sensor Scheduling for Aggregate Monitoring inWireless Sensor Networks" IEEE Computer Society, 19th International Conference on Scientific and Statistical Database Management (SSDBM 2007), 2007.

[5] Ossama Younis, Marwan Krunz, and Srinivasan Ramasubramanian,"Node Clustering in Wireless Sensor Networks: Recent Developments and Deployment Challenges", IEEE Network, pp. 20-25, May 2006.

[6] S. S. Kulkarni, "TDMA Services for Sensor Networks," Proc. 24th Int'l. Conf. Distrib. Comp. Sys. Wksps., Mar. 2004, pp. 604-09.

[7] Yanwei Wu, Xiang-Yang Li, YunHao Liu, and Wei Lou, "Energy-Efficient Wake-Up Scheduling for Data Collection and Aggregation" IEEE Transaction on Parallel and Distributed System, Vol. 21, No. 2, pp. 275-287, February 2010.

[8] Liqi Shi, and Abraham O. Fapojuwo, "TDMA Scheduling with Optimized Energy Efficiency and Minimum Delay in Clustered Wireless Sensor Networks", IEEE Transaction on Mobile Computing, Vol. 9, No. 7, pp. 927-939, July 2009.

[9] Bo Yu, Jianzhong Li, and Yingshu Li, "Distributed Data Aggregation Scheduling in Wireless Sensor Networks", in IEEE INFOCOM 2009, pp. 2159-2161, 2009.

[10] P.-J. Wan, K. M. Alzoubi, and O. Frieder, "Distributed construction of connected dominating set in wireless ad hoc networks," in INFOCOM, 2002.

[11] V. Rajendran, K. Obraczka, and J. J. Garcia-LunaAceves, "Energy-Efficient, Collision-Free Medium Access Control for Wireless Sensor Networks," Proc. ACM SenSys '03, Los Angeles, CA, Nov. 2003, pp. 181-92.

[12] G. Lu, B. Krishnamachari, and C. S. Raghavendra, "An Adaptive Energy-Efficient and Low-Latency MAC for Data Gathering in Wireless Sensor Networks," Proc. 18th Int'l. Parallel and Distrib. Processing Symp., Apr. 2004, p. 224. 